\title{
Kinetic energy functional for Fermi vapors in spherical harmonic confinement
}

\author{
A. Minguzzi, ${ }^{\text {a }}$ N. H. March ${ }^{\mathrm{b}, \mathrm{c}}$ and M. P. Tosi ${ }^{\mathrm{a}, *}$ \\ ${ }^{a}$ INFM and Classe di Scienze, Scuola Normale Superiore, 56126 Pisa, Italy \\ b Oxford University, Oxford, England \\ ${ }^{\mathrm{c}}$ Department of Physics, University of Antwerp (RUCA), Antwerp, Belgium
}

\begin{abstract}
Two equations are constructed which reflect, for fermions moving independently in a spherical harmonic potential, a differential virial theorem and a relation between the turning points of kinetic energy and particle densities. These equations are used to derive a differential equation for the particle density and a non-local kinetic energy functional.
\end{abstract}

Key words: Fermi gases, Density Functional Theory

PACS: 03.65.Db, 05.30.Fk, 71.10.Ca, 31.15.Ew

\section{Introduction}

There is currently considerable experimental activity in the area of harmonically confined fermion vapors $[1,2]$. This has been the motivation for the present Letter, in which equations are set up involving the particle density $\rho(r)$ and the kinetic energy density $t(r)$. We have written both quantities solely as functions of the radial distance $r$, as we shall be dealing with an arbitrary number, say $(M+1)$ closed shells in an isotropic external potential.

Two equations form the basis for what constitutes a density functional theory of independent harmonically confined fermions in three dimensions. The first is a form of differential virial equation, specific to an isotropic oscillator potential

$$
V(r)=\frac{1}{2} m \omega^{2} r^{2}
$$

\footnotetext{
* Corresponding author.
} 
where $\omega^{2}=k / m$, with $k$ the force constant and $m$ the fermion mass. The second equation relates the turning points of the particle density $\rho(r)$ and kinetic energy density $t(r)$, which we define from the wave function form

$$
t(r)=-\frac{\hbar^{2}}{2 m} \sum_{i} \psi_{i}(r) \nabla^{2} \psi_{i}(r) .
$$

Two other forms of the kinetic energy density will be used below, the first from the gradient of the wave function

$$
t_{G}(r)=\frac{\hbar^{2}}{2 m} \sum_{i}\left|\nabla \psi_{i}(r)\right|^{2}
$$

and the other derived as the average of $t(r)$ and $t_{G}(r)$,

$$
\bar{t}(r)=\frac{t(r)+t_{G}(r)}{2} .
$$

\section{Differential virial equation}

As to the differential virial equation mentioned above, the early work of March and Young [3], which however was restricted to one-dimensional motion along the $x$ axis, say, gave by expansion of the equation of motion of the Dirac density matrix around its diagonal:

$$
\frac{d t(x)}{d x}=-\frac{1}{2} \rho(x) \frac{d V(x)}{d x}-\frac{\hbar^{2}}{8 m} \rho^{\prime \prime \prime}(x) .
$$

This was termed a differential virial equation since, by multiplying by $x$ and integrating over all $x$ the usual integral virial theorem was recovered. We note from the definitions (2)-(4) above, with the consequence that

$$
t_{G}(\mathbf{r})=t(\mathbf{r})+\frac{\hbar^{2}}{4 m} \nabla^{2} \rho(\mathbf{r})
$$

that Eq. (5) can be rewritten

$$
\frac{d \bar{t}(x)}{d x}=-\frac{1}{2} \rho(x) \frac{d V(x)}{d x}
$$

While, for one dimension Eq. (7) is valid for a general confining potential $V(x)$, its generalization of present interest refers to a three-dimensional isotropic harmonic oscillator with closed-shells occupancy. It is not our purpose in this Letter to attempt any general proof, but we shall mention later a number of 


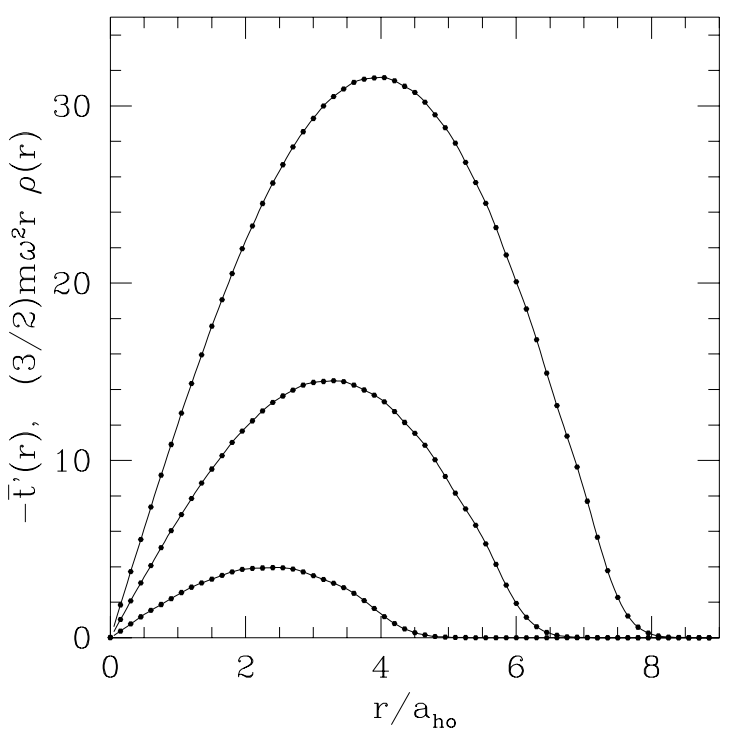

Fig. 1. Test of the differential virial equation: negative of $\partial \bar{t}(r) / \partial r$ (solid line) and $(3 / 2) m \omega^{2} r \rho(r)$ (dots) in units $\hbar \omega / a_{h o}^{4}$ as functions of the radial coordinate $r / a_{h o}$, for a 3D Fermi gas in isotropic harmonic confinement with 10, 20 and 30 filled shells (from bottom to top).

convincing confirmations of our result. This is

$$
\frac{\partial \bar{t}(r)}{\partial r}=-\frac{3}{2} \rho(r) \frac{\partial V(r)}{\partial r}
$$

but with $V(r)$ now restricted to the harmonic form (1). The factor $3 / 2$, by comparison with Eq. (7), is readily understood as due to dimensionality, as is confirmed by multiplying Eq. (8) throughout by $r$ to form the virial of the force $-\partial V(r) / \partial r$. A volume integration, followed by integration by parts on the LHS of the resulting equation yields

$$
2 T=\int d^{3} r \rho(r) r \frac{\partial V(r)}{\partial r}
$$

where $T=\int d^{3} r \bar{t}(r)$ is the total kinetic energy.

It is a very simple matter to verify Eq. (8) directly for the lowest shell only occupied, when $\psi_{M=0}(r)=A \exp \left(-r^{2} / 2 a_{h o}^{2}\right)$ and $\rho_{M=0}(r)=A^{2} \exp \left(-r^{2} / a_{h o}^{2}\right)$ with $a_{h o}=\sqrt{\hbar / m \omega}$ being the harmonic oscillator length and $A$ a normalization constant. More generally, numerical tests of Eq. (8) are shown in Fig. 1 for three values of the number of filled shells. In these calculations we have used the explicit expressions for the particle and kinetic energy densities reported by Brack and van Zyl [4] in terms of Laguerre polynomials.

As a last remark we note that, although the focus of the present work is on spherical harmonic confinement, any additional term that may enter the RHS 
of Eq. (8) for more general central $V(r)$ potentials does not contribute to the integral virial. Indeed Eq. (9) is the correct form of the virial theorem for such confinements.

\section{Turning points of particle and kinetic energy densities}

Having given these arguments for the form (8) of the differential virial equation for spherical harmonic confinement, we pass to the second central result of the present study, namely a relation between the turning points of kinetic energy and particle densities. Again, we can make a useful analogy with the onedimensional harmonic oscillator. With $N$ singly occupied shells we can write, following Lawes and March [5]:

$$
\frac{t^{\prime}(x)}{\rho^{\prime}(x)}=N \hbar \omega-\frac{1}{2} m \omega^{2} x^{2},
$$

the single lowest occupancy corresponding to $N=1$.

We now assert that, for the three-dimensional oscillator potential energy (1) the appropriate generalization of Eq. (10) reads

$$
\frac{t^{\prime}(r)}{\rho^{\prime}(r)}=(M+2) \hbar \omega-\frac{1}{2} m \omega^{2} r^{2}
$$

Again, no general proof will be attempted. But for the lowest shell $M=0$ only occupied, we can again use the explicit Gaussian wave function $\psi_{M=0}(r)$ given above to calculate $t(r)$ in Eq. (11) and $\rho_{M=0}^{\prime}(r)$ is also readily obtained. Equation (11) is confirmed for $M=0$ after a short calculation. More generally, Fig. 2 reports numerical tests of Eq. (11) for three values of the number of filled shells. Again the specific expressions of Brack and van Zyl [4] for $t(r)$ and $\rho(r)$ have been used.

Further confirmation, now in integral form but for general $M$, comes by multiplying Eq. (11) throughout by $r \rho^{\prime}(r)$ and doing a volume integration. Integration by parts readily yields, invoking the equipartition theorem for the harmonic oscillator,

$$
T=-(M+2) \hbar \omega \int_{0}^{\infty} r \rho^{\prime}(r) 4 \pi r^{2} d r
$$

But the integral on the RHS is related to the total number $N$ of fermions occupying $(M+1)$ closed shells, the simple result

$$
\frac{T}{N}=\frac{3}{8}(M+2) \hbar \omega
$$




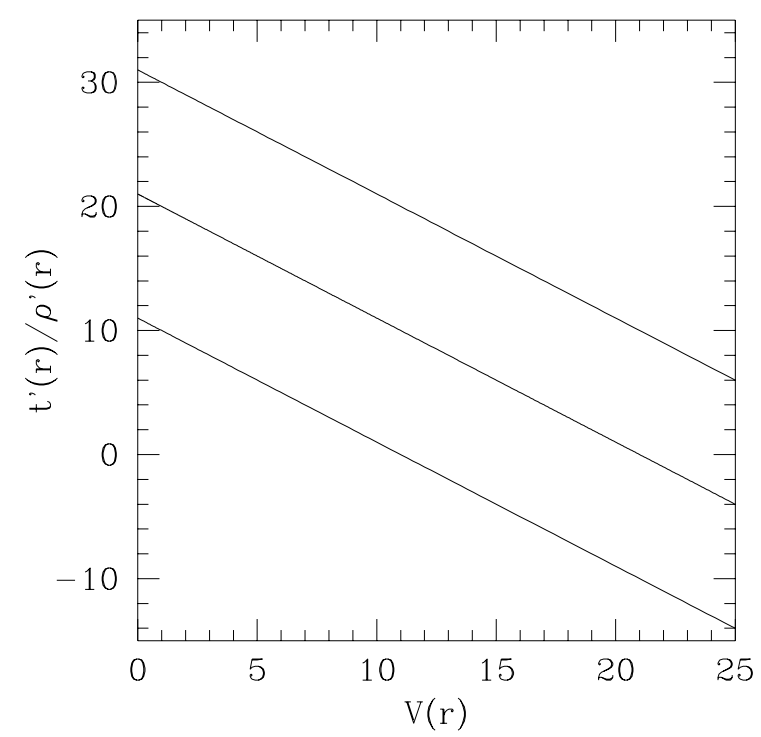

Fig. 2. Test of the relation between the turning points of particle and kinetic energy densities: $t^{\prime}(r) / \rho^{\prime}(r)$ in units $\hbar \omega$ as functions of the confining potential $V(r)=m \omega^{2} r^{2} / 2$, for a 3D Fermi gas in isotropic harmonic confinement with 10, 20 and 30 filled shells (from bottom to top).

following as a consequence of Eq. (11). This is readily confirmed by calculating the eigenvalue sum $E$ from the oscillator levels plus the known degeneracies. Again, for $N=1$ or equivalently $M=0, T=3 \hbar \omega / 4$ follows immediately from the virial result $T=E / 2$ since $E=3 \hbar \omega / 2$ for the lowest shell.

Before considering the consequences of Eq. (11), when combined with the differential virial theorem, we want to stress that, for $M>0$, the Pauli Exclusion Principle plays a role in determining both particle and kinetic energy densities. Therefore, as a further confirmation of Eq. (11) we have used explicit harmonic oscillator wave functions (see e.g. Morse and Feshbach [6]) to construct the Dirac density matrix $\gamma\left(\mathbf{r}, \mathbf{r}^{\prime}\right)$ for two shells occupied, i.e. for $M=1$. Since the degeneracy of the levels for a given total quantum number $n$ is $(n+1)(n+2) / 2$, one is then dealing for singly occupied levels with 4 particles, and the Dirac matrix is readily constructed as

$$
\gamma_{M=1}\left(\mathbf{r}, \mathbf{r}^{\prime}\right)=\left(\pi^{1 / 2} a_{h o}\right)^{-3}\left[1+2\left(x x^{\prime}+y y^{\prime}+z z^{\prime}\right) / a_{h o}^{2}\right] \exp \left[-\left(r^{2}+r^{\prime 2}\right) / 2 a_{h o}^{2}\right] .
$$

Forming the kinetic energy density from

$$
t_{M=1}(r)=-\left.\frac{\hbar^{2}}{2 m} \nabla_{\mathbf{r}^{\prime}}^{2} \gamma_{M=1}\left(\mathbf{r}, \mathbf{r}^{\prime}\right)\right|_{\mathbf{r}^{\prime}=\mathbf{r}}
$$

and using the explicit form of $\rho^{\prime}(r)$ following from differentiating with respect to $r$ the diagonal density $\rho_{M=1}(r)=\left.\gamma_{M=1}\left(\mathbf{r}, \mathbf{r}^{\prime}\right)\right|_{\mathbf{r}^{\prime}=\mathbf{r}}$, once again confirms the correctness of Eq. (11) for $t^{\prime}(r) / \rho^{\prime}(r)$. 


\section{Density functional theory}

Given therefore Eqs. (8) and (11) as the two pillars of the present study we go on to construct the density functional theory of this problem of threedimensional harmonic confinement. First of all, let us use Eqs. (4) and (6) to remove $\bar{t}(r)$ in Eq. (8) in favour of $t(r)$ and $\nabla^{2} \rho(r)$. Then invoking Eq. (11) the derivative $\partial t(r) / \partial r$ can be eliminated, the result being a differential equation for the particle density $\rho(r)$. This reads

$$
\frac{\hbar^{2}}{8 m} \frac{\partial}{\partial r}\left[\nabla^{2} \rho(r)\right]+[(M+2) \hbar \omega-V(r)] \rho^{\prime}(r)+\frac{3}{2} \frac{\partial V(r)}{\partial r} \rho(r)=0
$$

which we must again emphasize is valid for the three-dimensional potential energy (1). Equation (16) is evidently a third-order, linear, homogeneous equation for the particle density $\rho(r)$ of $(M+1)$ closed shells. Since the pioneering work of von Weizsäcker [7], it has been clear that a major objective of density functional theory is to construct directly the fermion particle density from a given one-body potential. Equation (16) achieves that aim quite explicitly for closed shells in the harmonic potential (1). It is the three-dimensional generalization of the early result of Lawes and March [5] for one-dimensional harmonic confinement.

But there is a further consequence of the two basic equations (8) and (11). For one can alternatively eliminate the potential, in order to directly relate kinetic energy and particle densities. One finds almost immediately

$$
\frac{\partial \bar{t}(r)}{\partial r}=\frac{3}{2} \rho(r) \frac{\partial}{\partial r}\left[\frac{t^{\prime}(r)}{\rho^{\prime}(r)}\right]
$$

As emphasized earlier, $\bar{t}(r)$ and $t(r)$ differ only by a constant times the Laplacian of the particle density. The resulting relation can be integrated, using an appropriate integrating factor, to give $t(r)$ in terms of integrals of $\rho(r)$ and its low-order derivatives. Explicitly we find

$$
\frac{\partial}{\partial r}\left[\frac{t^{\prime}(r)}{\rho^{\prime}(r) \rho^{2 / 3}(r)}\right]=\frac{\hbar^{2}}{12 m} \frac{1}{\rho^{5 / 3}(r)} \frac{\partial}{\partial r} \nabla^{2} \rho(r)
$$

One can reduce the order of the differential equation (18) by explicit integration and by using the "boundary condition" $\left[t^{\prime}(r) / \rho^{\prime}(r)\right]_{r=0}=(M+2) \hbar \omega$ from Eq. (11) to obtain

$$
t^{\prime}(r)=\frac{(M+2) \hbar \omega}{\rho^{2 / 3}(0)} \rho^{2 / 3}(r) \rho^{\prime}(r)+\frac{\hbar^{2}}{12 m} \rho^{2 / 3}(r) \rho^{\prime}(r) \int_{0}^{r} d s \frac{1}{\rho^{5 / 3}(s)} \frac{\partial}{\partial s} \nabla^{2} \rho(s) .
$$


A second integration leads to the final result

$$
t(r)=\frac{t_{W}(r)}{3}+\left\{C+\frac{\hbar^{2}}{3 m} \int_{0}^{r} d s \frac{\left[\rho^{\prime}(s)\right]^{2}}{\rho^{8 / 3}(s)}\left[\frac{1}{2 s}+\frac{\rho^{\prime}(s)}{3 \rho(s)}\right]\right\} \rho^{5 / 3}(r)
$$

where $t_{W}(r)=\left(\hbar^{2} / 8 m\right)\left[\rho^{\prime}(r)\right]^{2} / \rho(r)$ is the von Weizsäcker "surface" contribution to the kinetic energy density [7] and the constant $C$ is given by $C=3(M+2) \hbar \omega /\left(5 \rho^{2 / 3}(0)\right)-\left(\hbar^{2} / 20 m\right)\left(\nabla^{2} \rho(r)\right)_{r=0} / \rho^{5 / 3}(0)$. We have assumed $\partial \rho(r) /\left.\partial r\right|_{r=0}=0$.

The form $\rho^{5 / 3}(r)$ in Eq. (20) suggests, with of course the exact density $\rho(r)$, the introduction of the Thomas-Fermi (TF) kinetic energy density defined by

$$
t_{T F}(r)=c_{k} \rho^{5 / 3}(r) .
$$

The constant $c_{k}$ is a multiplicative factor which we do not need to specify for the present purposes. Thus, as in earlier work in this Journal [8] on the onedimensional case of harmonic confinement, we obtain that $t_{T F}(r)$ and $t_{W}(r)$ enter as building blocks the non-local kinetic energy density functional (20).

\section{Summary}

In summary, we have derived two relatively simple equations, namely Eqs. (8) and (11), the first being a differential form of the virial theorem and the second relating turning points of kinetic energy density $t(r)$ and particle density $\rho(r)$. These allow (i) the differential equation (16) for $\rho(r)$ and (ii) the non-local kinetic energy density $t(r)$ in Eq. (20) to be obtained.

\section{Acknowledgements}

This work was partially supported by MURST through PRIN2000. N.H.M. wishes to acknowledge generous support from SNS for a stay in Pisa during which much of his contribution to the present work was carried out. N.H.M. also acknowledges valuable discussions with Dr. T. Gál and with Professors L. C. Balbás, A. Holas and Á. Nagy on the general area embraced by the present investigation.

\section{References}


[1] B. DeMarco and D. S. Jin, Science 285 (1999) 1703; M. J. Holland, B. DeMarco, and D. S. Jin, Phys. Rev. A 61 (2000) 053610.

[2] M. O. Mewes, G. Ferrari, F. Schreck, A. Sinatra, and C. Salomon, Phys. Rev. A 61 (2000) 011403(R); F. Schreck, G. Ferrari, K. L. Corwin, G. Cubizolles, L. Khyakovich, M. O. Mewes, and C. Salomon, cond-mat/0011291.

[3] N. H. March and W. H. Young, Nucl. Phys. 12 (1959) 237.

[4] M. Brack and B. P. van Zyl, cond-mat/0010201.

[5] G. P. Lawes and N. H. March, J. Chem. Phys. 71 (1979) 1007.

[6] P. M. Morse and H. Feshbach, Methods of Theoretical Physics (MacGraw-Hill, New York, 1953).

[7] C. F. von Weizsäcker, Z. Phys. 96 (1935) 431.

[8] N. H. March, P. Senet, and V. E. Van Doren, Phys. Lett. A 270 (2000) 88. 